\title{
Urinary Exosomal Long Noncoding RNA TERC as a Noninvasive Diagnostic and Prognostic Biomarker for Bladder Urothelial Carcinoma
}

\author{
Chen Chen $\mathbb{D}^{1},{ }^{1}$ Anquan Shang $\mathbb{D}^{1},{ }^{1}$ Zujun Sun $\mathbb{D}$, ${ }^{1}$ Yuting Gao $\mathbb{D},{ }^{1}$ Jingjuan Huang $\mathbb{D}$, ${ }^{1}$ \\ Yili Ping $\mathbb{D}$, ' Wenjing Chang $\mathbb{D}^{1},{ }^{1}$ Chenzheng Gu $\mathbb{D}^{1},{ }^{1}$ Junjun Sun $\mathbb{D}^{1},{ }^{1}$ Ping Ji $\mathbb{D}^{1},{ }^{1}$ Yi Yuan $\mathbb{D}^{1}{ }^{1}$ \\ Renquan Lu $(\mathbb{D})^{2,3}$ and Dong $\mathrm{Li} \mathbb{D}^{1}$ \\ ${ }^{1}$ Department of Laboratory Medicine, Shanghai Tongji Hospital, School of Medicine, Tongji University, Shanghai 200065, China \\ ${ }^{2}$ Department of Clinical Laboratory, Fudan University Shanghai Cancer Center, Shanghai, China \\ ${ }^{3}$ Department of Oncology, Shanghai Medical College, Fudan University, Shanghai, China
}

Correspondence should be addressed to Renquan Lu; renquanlu@fudan.edu.cn and Dong Li; lidong@tongji.edu.cn

Received 1 November 2021; Accepted 23 December 2021; Published 25 January 2022

Academic Editor: Wagner Batista

Copyright (c) 2022 Chen Chen et al. This is an open access article distributed under the Creative Commons Attribution License, which permits unrestricted use, distribution, and reproduction in any medium, provided the original work is properly cited.

\begin{abstract}
Purpose. Bladder cancer is one of the most common urological malignancies worldwide, and approximately $90 \%$ of bladder cancer cases are histologically typed as bladder urothelial carcinoma (BLCA). Exosomes are 30 to $200 \mathrm{~nm}$ extracellular vesicles that transport microRNAs, long noncoding RNAs (lncRNAs), mRNAs, circular RNAs, and proteins across tissues and through circulation. Urinary exosomes may contain genetic information from tumor cells. Herein, we explored the clinical significance of urinary exosomal lncRNA telomerase RNA component (TERC) levels to provide an urgently needed diagnostic and prognostic biomarker for BLCA. Materials and Methods. In this study, we used RNA-sequencing of samples from four BLCA patients and three healthy controls to identify that TERC was differentially expressed in urinary exosomes. We then used quantitative PCR in different types of clinical samples to validate the biomarker and analyzed results using receiver operating characteristic curves. Results. We found that TERC was significantly upregulated in urinary exosomes from BLCA patients compared with those from healthy controls $(P<0.0001)$. Urinary exosomal TERC showed higher sensitivity $(78.65 \%)$ and accuracy $(77.78 \%)$ than existing indicators including nuclear matrix protein-22 and urine cytometry. Using the cut-off value 4.302, the area under the curve for urinary exosomal TERC was 0.836 (95\% confidence interval: $0.768-0.891, P<0.0001$ ). Furthermore, this noninvasive assay could distinguish low-grade and high-grade tumors $(P=0.0153)$. Conclusions. TERC is enriched in urinary exosomes from BLCA patients. Urinary exosomal TERC could become a diagnostic and prognostic biomarker for BLCA that allows clinicians to realize noninvasive detection of BLCA.
\end{abstract}

\section{Introduction}

Globally, bladder cancer is one of the most common urological malignancies, with approximately $90 \%$ of cases histologically defined as bladder urothelial carcinoma (BLCA). In 2020, the incidence of bladder cancer ranked tenth among all malignant tumors, and its incidence and mortality ranked second among male urinary system malignancies [1]. The gold standard for diagnosing bladder cancer remains tissue biopsy via cystoscopy. The colloidal gold immunochromatographic assay (GICA) for nuclear matrix protein- (NMP-)
22 and bladder tumor antigen are clinical methods approved by the US Food and Drug Administration; however, their sensitivity is too low, and hematuria always leads to false positive results [2-4]. Thus, there is an urgent need for a more meaningful diagnostic index for bladder cancer that balances sensitivity and specificity.

Exosomes are potential diagnostic samples from liquid biopsy because they can transport mRNAs, microRNAs, long noncoding RNAs (lncRNAs), circular RNAs, lipids, and proteins throughout bodily fluids $[5,6]$. The exosomal lipid bilayer protects the contents from RNases and 
proteases [7]. IncRNAs are defined as transcripts of $>200$ nucleotides and have been demonstrated to play critical roles in many biological and pathological processes rather than being "transcriptional noise" [8]. It has been reported that exosomes can selectively package certain lncRNAs that are abundantly present in exosomes $[9,10]$. BLCA tumor cells continuously release exosomes that contain lncRNAs into urine. Urinary exosomal lncRNAs can directly reflect the malignant state and have the possibility to be diagnostic and prognostic biomarkers for BLCA [11-14].

Using deep RNA-sequencing of urinary exosomes, we found that the IncRNA telomerase RNA component (TERC, $451 \mathrm{nt}$ in length) was differently expressed between four BLCA patients and three healthy controls. TERC and telomerase reverse transcriptase (TERT) compose telomerase. In most human tumors, telomerase is activated to maintain telomere length, extend lifespan, and reduce apoptosis of tumor cells [15]. Many previous studies have reported the roles of TERC in telomere biology and in promoting tumorigenesis and inflammation, but there are still few reports regarding the diagnostic and/or prognostic value of exosomal TERC expression [16-19].

\section{Materials and Methods}

2.1. Patients and Volunteers. Samples were collected from BLCA patients with histopathologically confirmed diagnosis between October 2019 and May 2021 at Shanghai Tongji Hospital and Fudan University Shanghai Cancer Center. The clinical information of patients is provided in Supplementary File 1. In total, healthy participants were sought. The Ethics Committee of Shanghai Tongji Hospital (No. 2021-KYSB-064) and Fudan University Shanghai Cancer Center (No. 050432-4-1911D) authorized this study.

2.2. Isolation of Exosomes. Exosomes for sequencing were isolated from $150 \mathrm{~mL}$ of urine using differential centrifugation. The isolation procedure was as follows, taking the supernatant from each step: urine was centrifuged at $600 \times$ $g$ for $20 \mathrm{~min}, 2000 \times g$ for $30 \mathrm{~min}$, and $10000 \times g$ for $1 \mathrm{~h}$ and then filtered through a $0.22 \mu \mathrm{m}$ filter. Finally, ultracentrifugation at $100000 \times g$ for $2 \mathrm{~h}$ was performed, and the supernatant was discarded. All centrifugation steps were performed at $4^{\circ} \mathrm{C}$. Exosome pellets were resuspended in $50 \mu \mathrm{L}$ of $0.22 \mu \mathrm{m}$ filtered phosphate-buffered saline.

Exosomes used for validation were isolated from human urine in strict accordance with the instructions of the exosome extraction kit (BestBio, Shanghai, China). $8 \mathrm{~mL}$ of urine was centrifuged at $3000 \times g$ for $15 \mathrm{~min}$ at $4^{\circ} \mathrm{C}$, and the supernatant was then centrifuged at $10000 \times g$ for $20 \mathrm{~min}$ at $4^{\circ} \mathrm{C}$. The supernatant was then mixed with solution $\mathrm{A}$ and stored $4^{\circ} \mathrm{C}$ overnight. The mixture was centrifuged at $10000 \times g$ for $1 \mathrm{~h}$ at $4^{\circ} \mathrm{C}$ and exosome pellets were resuspended in $500 \mu \mathrm{L}$ TRIzol-LS (Life Technologies, Carlsbad, CA, USA).

2.3. Transmission Electron Microscopy (TEM). Exosome suspensions were placed on 400-mesh carbon-coated copper grids and negatively stained with $2 \%$ phosphotungstic acid
TABle 1: Primer sequences are listed in the table.

\begin{tabular}{lcc}
\hline \multirow{2}{*}{ TERC } & Forward & $5^{\prime}$-GTGGTGGCCATTTTTTGTCTAAC-3 \\
& Reverse & $5^{\prime}$-TGCTCTAGAATGAACGGTGGAA-3' \\
& Forward & $5^{\prime}$-TGATGACATCAAGAAGGTGG-3' \\
GAPDH & Reverse & $5^{\prime}$-TTGTCATACCAGGAAATGAGC-3' \\
& Forward & $5^{\prime}$-CGTTCTTAGTTGGTGGAGCG-3' \\
18S & Reverse & $5^{\prime}$-CGCTGAGCCAGTCAGTGTAG-3
\end{tabular}

solution. TEM was performed to view and capture images (Thermo Fisher Scientific, Waltham, MA, USA).

2.4. Nanoparticle Tracking Analysis (NTA). The NanoSight LM10 system (Malvern Instruments Ltd., Malvern, UK) was used to detect the concentration and size distribution of particles.

2.5. Western Blot Analysis. Exosome suspensions were mixed with the appropriate volume of $5 x$ SDS loading buffer, the mixture was boiled for $15 \mathrm{~min}$ at $100^{\circ} \mathrm{C}$, and then, the supernatant was collected for western blot analysis. The primary antibodies included anti-TSG101 (Abcam, Cambridge, UK), anti-HSP70, anti-Annexin V, and anti-CD9 (all from Cell Signaling Technology, Danvers, MA, USA). The secondary antibody used was peroxidase-conjugated goat antirabbit IgG (Millipore, Burlington, MA, USA).

2.6. RNA-Sequencing. RNA-sequencing (RNA-seq) was performed by Shanghai Kangcheng Biotechnology Company (Shanghai, China) using an Illumina Novaseq 6000 system (San Diego, CA, USA). Differences in transcript levels were analyzed and used to screen out the differentially expressed lncRNAs between the BLCA patient and healthy control groups. The comparison scheme was fold change $>1.5, P$ value $(F$ test $) \leq 0.05$, and mean $F P K M \geq 0.5$ in each sample.

2.7. RNA Extraction and Real-Time Quantitative PCR (qPCR). TRIzol-LS reagent was used to extract total exosomal RNA, cDNA was synthesized using the PrimeScript $^{\mathrm{TM}}$ RT reagent kit with gDNA Eraser (Takara, Dalian, Japan), and qPCR was performed using the TB Green Premix Ex Taq II (Tli RNaseH Plus) (Takara) on an Applied Biosystems 7300 real-time PCR system (Waltham, MA, USA). Table 1 shows the sequences of primers used in this study.

2.8. GICA and Enzyme-Linked Immunosorbent Assay (ELISA) Analyses of NMP-22. Urine exosomal NMP-22 was detected using the Alere NMP22 ${ }^{\circledR}$ BladderChek ${ }^{\circledR}$ Test based on GICA and ELISA kits from Hualian Biotechnology (Wuhan, China). The assays were performed in accordance with the kit instructions.

2.9. Statistical Analysis. Data are presented as mean \pm SD. The Mann-Whitney $U$ test, Pearson's $\chi^{2}$ test, and Pearson's correlation analysis were used to assess differences or 


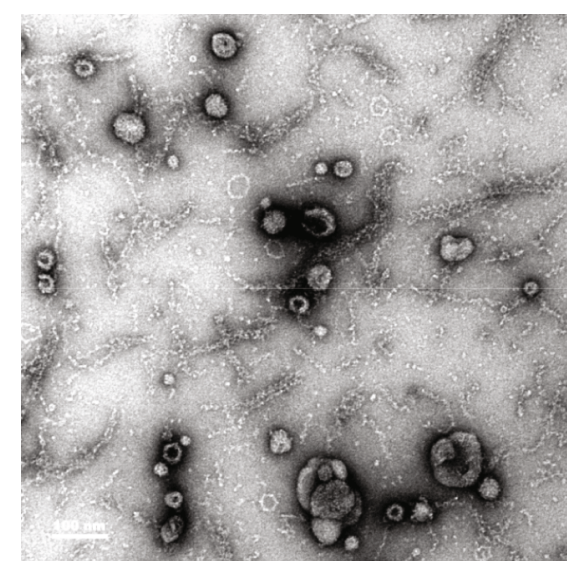

(a)

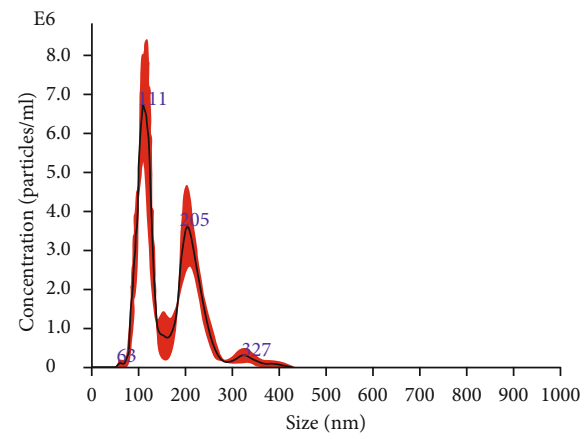

(b)

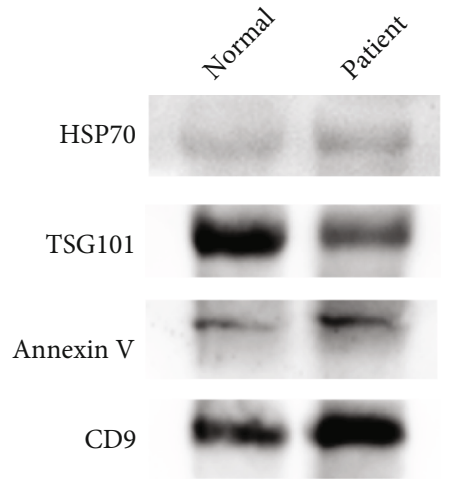

(c)

Figure 1: Characterization of urinary exosomes. (a) Transmission electron microscopy image of exosomes isolated from urine; scale bar: $100 \mathrm{~nm}$. (b) Particles were observed in the size range of 50 to $200 \mathrm{~nm}$ by nanoparticle tracking analysis. (c) Western blot analysis of the exosomal protein markers HSP70, TSG101, Annexin V, and CD9. Exosomes were derived from healthy controls and bladder urothelial carcinoma patients.

correlation. $P<0.05$ was considered statistically significant. Receiver operating characteristic (ROC) curves were used to determine the sensitivity and specificity of indicators. All statistical analyses were performed using GraphPad Prism 8 (GraphPad Software, Inc., Sand Diego, CA, USA) and MedCalc version 15.2.2 (MedCalc Software Ltd., Ostend, Belgium). Analysis of Kaplan-Meier survival curves was performed using data from the PrognoScan database for BLCA (GSE13507).

\section{Results}

3.1. Identification of Exosomes Derived from Urine Samples. According to the 2018 checklist for minimum information for studies of extracellular vesicles (MISEV2018) [20], we used a combination of TEM, NTA, and western blot analysis to identify exosomes. TEM showed that urinary exosomes were of round shape with cup-like concavity (Figure 1(a)). The size distributions of exosomes were analyzed by NTA. As shown in Figure 1(b), the size (diameter) of urinary exosomes ranged from approximately 50 to $200 \mathrm{~nm}$ (mean: $165.2 \mathrm{~nm}$ ). Positive expression of four exosomal protein markers was confirmed by western blot analysis (Figure 1(c)) including of two cytosolic proteins (TSG101 and Annexin V) and two transmembrane proteins (HSP70 and CD9). Only strictly identified urine exosomes were used for further analysis and research [21].

3.2. RNA-Sequencing of Urinary Exosomes. To identify differentially expressed BLCA-specific molecules, we performed RNA-sequencing on urine exosomes from four BLCA patients and three healthy controls [22]. The results revealed 106 differentially expressed lncRNAs between urine exosomes from BLCA patients and healthy donors, among which 103 were upregulated and three were downregulated (Figures 2(a) and 2(b)). Among the upregulated lncRNAs, we found high abundance of TERC, which has been previously reported to be related to "tumor growth."
3.3. Increased TERC Expression in Urine Exosomes from BLCA Patients. We performed $\mathrm{qPCR}$ to evaluate relative TERC expression in different types of clinical samples. We found that $18 \mathrm{~S}$ levels were more stable and abundant in urinary exosomes than the conventional housekeeping gene $G A P D H$ and was more suitable as an internal reference gene for lncRNAs in exosomes. The absolute expression levels of genes are listed in Supplementary File 2. First, we detected TERC expression in urine exosomes from the three healthy controls and four BLCA patients used for sequencing (Figure 3(a)). In this small sample, we verified that TERC expression was higher in urinary exosomes from BLCA patients than from healthy controls, which was consistent with the RNA-sequencing results.

TERC was then detected in urine exosomes from 94 healthy controls, 46 patients with urinary benign lesions, and 128 BLCA patients (Figure 3(c)). The findings suggested that urinary exosomal TERC expression in BLCA patients was significantly higher compared with that in healthy controls and patients with urinary benign lesions $(P<0.0001)$, indicating that urinary exosomal TERC may have diagnostic value for BLCA. As shown in Figure 3(b), there was no difference between TERC expression of tumors $(n=39)$ and that of adjacent tissues $(n=23)$.

3.4. Urinary Exosomal TERC Had Better Diagnostic Value than NMP-22 (ELISA and GICA) and Urine Cytology. We examined TERC levels in urine exosomes from 89 BLCA patients and 63 healthy controls to determine its diagnostic utility. ROC curve analysis using the cut-off value 4.302 showed that TERC was a promising diagnostic biomarker with an area under the curve (AUC) of 0.836 (95\% confidence interval [CI]: $0.768-0.891, P<0.0001)$. The sensitivity and specificity of TERC were $78.65 \%$ and $77.78 \%$, respectively (Figure 4(a)). Meanwhile, we detected NMP-22 from urine samples using ELISA; the results are listed in Supplementary File 2. The AUC, sensitivity, and specificity of NMP-22 (ELISA) were 0.696 (95\% CI: $0.616-0.768, P<$ 


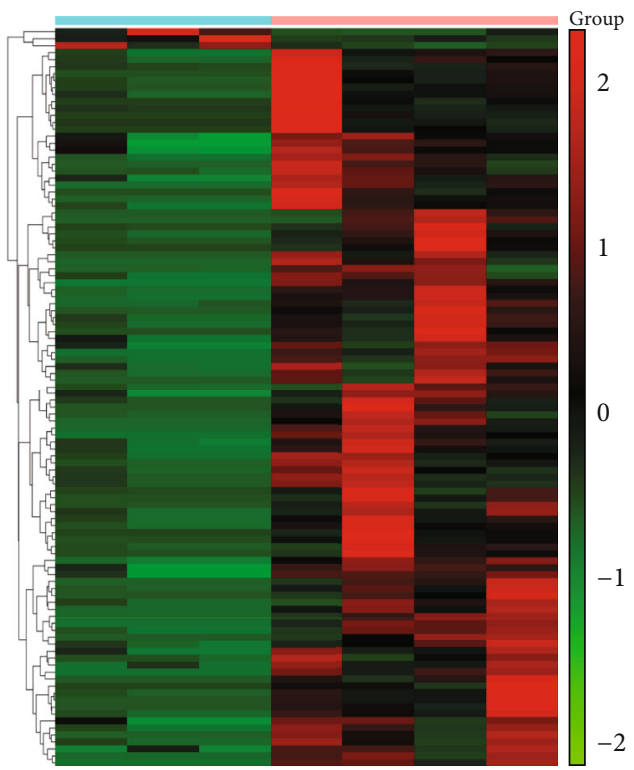

(a)

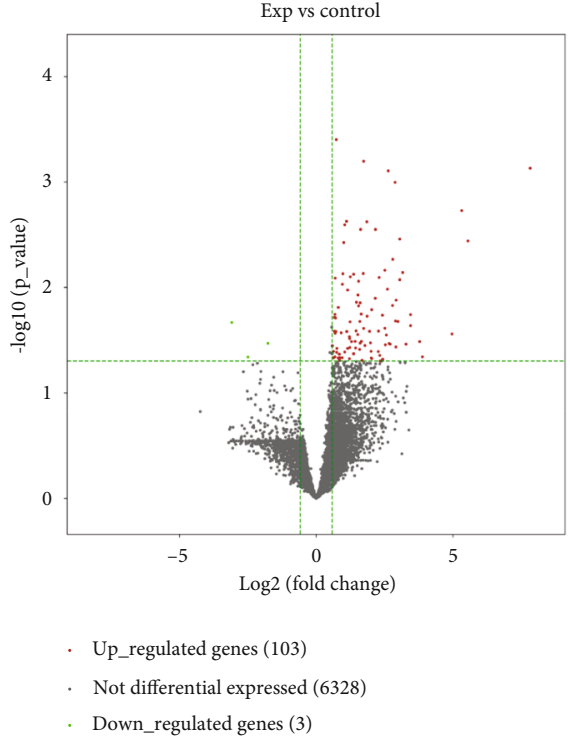

(b)

FIGURE 2: Heat map and volcano plot of differentially expressed long noncoding RNAs (DE-lncRNAs) from urinary exosomes. (a) Heat map of all DE-lncRNAs from urinary exosomes of healthy controls and bladder urothelial carcinoma (BLCA) patients. Results of the differential analysis are shown $(P \leq 0.05)$, with color from green to red indicating low to high fragments per kilobase of exon per million mapped fragments (FPKM) of DE-lncRNAs. (b) Volcano plot of RNA-sequencing results. Green dots represent downregulated genes and red dots represent upregulated genes.

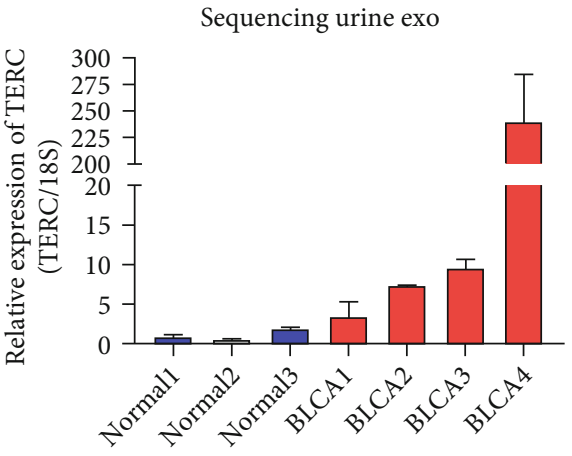

(a)

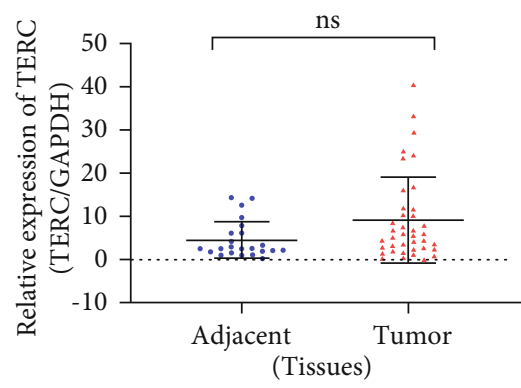

- Adjacent

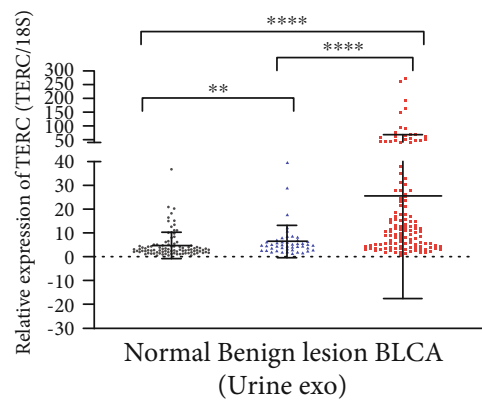

Normal Benign lesion BLCA

FIgURE 3: Relative telomerase RNA component (TERC) expression levels in bladder urothelial carcinoma (BLCA) patients. (a) TERC expression levels in urinary exosome samples from the three healthy controls and four BLCA patients used for RNA-sequencing are shown as mean \pm SD. (b, c) Real-time quantitative PCR detection of TERC in different sample types including urine exosomes and tissues (ns: $P>0.05,{ }^{* *} P<0.01,{ }^{* * * *} P<0.0001$ ). Relative expression levels in tissues were normalized to GAPDH, while $18 S$ was used as the internal reference gene in exosomes. Groups were compared with the nonparametric Mann-Whitney $U$ test.

0.0001 ), 60.67\%, and 74.6\%, respectively (Figure 4(b)). The AUC of combining the indicators reached 0.861 (95\% CI: 0.795-0.911) (Figure 4(c)). Additionally, we analyzed NMP-22 (GICA) and urine cytology in 128 patients and 94 healthy controls. The results illustrated the high specificities of NMP-22 (GICA) and urine cytology (96.809\% and
$98.936 \%$, respectively), but their sensitivities $(31.25 \%$ and $43.75 \%$, respectively) were much lower than that of urine exosomal TERC (78.65\%) (Tables 2 and 3). In summary, these results demonstrated that urinary exosomal TERC had better diagnostic abilities than the existing indicators (NMP-22 [ELISA and GICA] and urine cytology). 


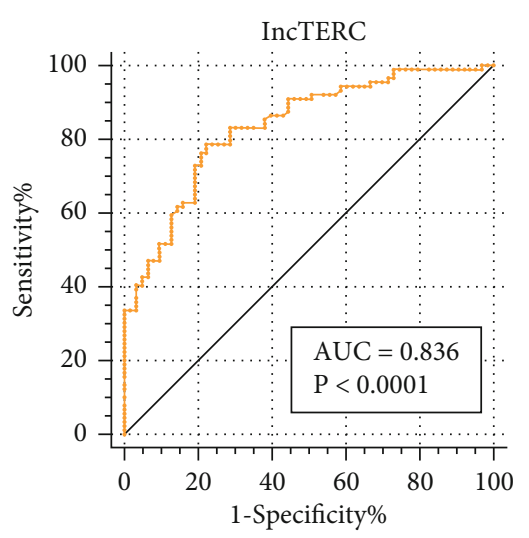

(a)

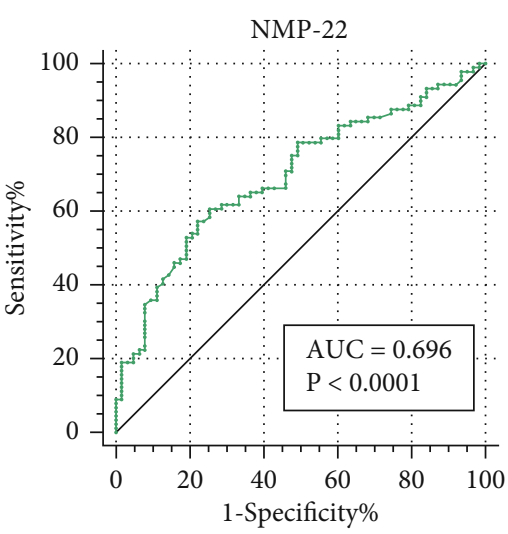

(b)

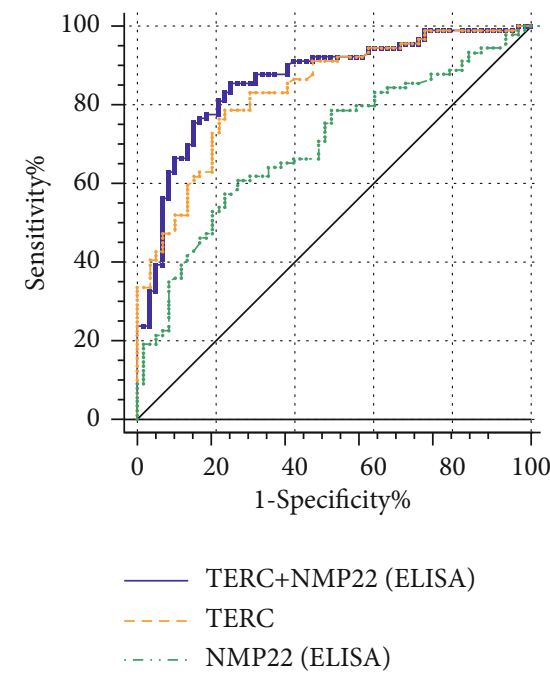

(c)

FIGURE 4: Diagnostic ability of exosomal telomerase RNA component (TERC) and nuclear matrix protein- (NMP-) 22 for bladder urothelial carcinoma (BLCA). (a-c) Receiver operating characteristic curve analysis of exosomal TERC and NMP-22 (ELISA) and the combination of these indicators revealed area under the curve values of $0.836,0.696$, and 0.861 .

TABLE 2: Statistics of NMP-22 (GICA) and urine cytology in BLCA patients and normal individuals.

\begin{tabular}{lccc}
\hline & & BLCA & Normal \\
\hline \multirow{2}{*}{ NMP-22 (GICA) } & Positive & 40 & 3 \\
& Negative & 88 & 91 \\
\multirow{2}{*}{ Urine cytology } & Positive & 56 & 1 \\
& Negative & 72 & 93 \\
\hline
\end{tabular}

TABle 3: Diagnostic efficacy of NMP-22 (GICA) and urine cytology.

\begin{tabular}{lcc}
\hline & NMP-22 (GICA) & Urine cytology \\
\hline Sensitivity & $31.250 \%$ & $43.750 \%$ \\
Specificity & $96.809 \%$ & $98.936 \%$ \\
Positive likelihood ratio & 9.792 & 41.125 \\
Negative likelihood ratio & 0.710 & 0.569 \\
Positive predictive value & $93.023 \%$ & $98.246 \%$ \\
Negative predictive value & $50.838 \%$ & $56.364 \%$ \\
\hline
\end{tabular}

3.5. Urine Exosomal TERC Was Associated with Tumor Grade, and Bioinformatics Analysis Showed Prognostic Predictive Value for BLCA. Patients' clinical characteristics including age, sex, TNM stage, histological grade, metastasis, and recurrence are summarized in Table 4. Pearson's $\chi^{2}$ test was performed to explore associations between exosomal TERC levels and clinical pathological characteristics. Patients were divided into the low$(n=64)$ and high-expression $(n=64)$ groups, using the median value of TERC expression as the cut-off value. The findings indicated that the level of TERC expression was associated with tumor grade $(P=0.0153)$, while other clinical features such as metastasis and recurrence were not significantly correlated with TERC. Thus, we conclude that high TERC expression is associated with BLCA disease progression. Meanwhile, we analyzed Kaplan-Meier curves of disease-specific survival (DSS) and overall survival (OS) according to TERC expression using the PrognoScan database (http://dna00.bio.kyutech.ac.jp/ PrognoScan/index.html) based on GSE13507. The hazard 
TABLE 4: The characteristics of bladder urothelial carcinoma patients.

\begin{tabular}{|c|c|c|c|c|c|}
\hline \multirow[t]{2}{*}{ Variables } & \multirow[t]{2}{*}{ Total } & \multicolumn{2}{|c|}{$\begin{array}{c}\text { Expression } \\
\text { of TERC }\end{array}$} & \multirow[t]{2}{*}{$\chi^{2}$} & \multirow[t]{2}{*}{$P$ value } \\
\hline & & High & Low & & \\
\hline Age & & & & 0.82 & 0.3651 \\
\hline$\leq 60$ & 49 & 22 & 27 & & \\
\hline$>60$ & 79 & 42 & 37 & & \\
\hline Sex & & & & 3.099 & 0.0783 \\
\hline Male & 98 & 44 & 54 & & \\
\hline Female & 30 & 19 & 11 & & \\
\hline TNM & & & & 1.315 & 0.2515 \\
\hline $\mathrm{Ta} \sim \mathrm{T} 1$ & 105 & 50 & 55 & & \\
\hline $\mathrm{T} 2 \sim \mathrm{T} 4$ & 23 & 14 & 9 & & \\
\hline Grade & & & & 5.88 & $0.0153^{\mathrm{a}}$ \\
\hline Low & 20 & 5 & 15 & & \\
\hline High & 108 & 59 & 49 & & \\
\hline Lymphatic metastasis & & & & 1.026 & 0.3110 \\
\hline Negative & 110 & 53 & 57 & & \\
\hline Positive & 18 & 11 & 7 & & \\
\hline Recurrence & & & & 1.233 & 0.2668 \\
\hline Primary & 103 & 54 & 49 & & \\
\hline Recurrent & 25 & 10 & 15 & & \\
\hline
\end{tabular}

ratios (HRs) for DSS and OS were 1.79 and 1.51, respectively (Figures 5(a) and 5(b)). These results indicated that urinary exosomal TERC was a significant risk predictor with the potential to be a prognostic biomarker for BLCA.

${ }^{\mathrm{a}} P<0.05$, Pearson's $\chi^{2}$ test for the variables above in patients.

3.6. Urine Exosomal TERC Expression Was Not Correlated with Paired Tissues, and It Stably Exists after Freeze-Thaw Cycles due to Protection from the Lipid Bilayer. To investigate the relationship in TERC expression between different types of samples, TERC levels were measured by qPCR in paired tissues and urine exosomes from BLCA patients [23]. Pearson's correlation analysis demonstrated that urine exosomal TERC levels were not correlated with the TERC levels in corresponding tissues; the correlation coefficients $(r)$ were 0.00954 (Figure 6(a)).

Additionally, we divided urine samples into five aliquots of equal volume and placed each at $-80^{\circ} \mathrm{C}$ for one to five freeze-thaw cycles [24]. The results revealed that exosomal TERC levels were stable after multiple freeze-thaw cycles (Figure 6(b)). This suggested that storing urine at $-80^{\circ} \mathrm{C}$ even with multiple freeze-thaw cycles will not affect exosomal TERC, which was superior to detecting TERC in urine sediment cells $[25,26]$. In summary, urinary exosomal TERC has the potential to be a stable and noninvasive diagnostic and prognostic biomarker for BLCA.

\section{Discussion}

Bladder cancer is one of the most frequent malignancies of the urinary system, with $90 \%$ of cases pathologically classified as BLCA. The cause of BLCA tumorigenesis is currently unclear and may include smoking, repeated infection, exposure to compounds, or genetic factors [27, 28]. The gold standard for diagnosing bladder cancer is pathological tissue biopsy, which is invasive and uncomfortable. This study introduced a novel assay that does not require expedited or other special handling procedures, and an $8 \mathrm{~mL}$ urine sample is more than enough for the analysis.

We identified that the IncRNA TERC was differentially upregulated in urinary exosomes of BLCA patients by RNA-sequencing. The important components of the telomerase are TERT and TERC. As previously reported, identifying TERT promoter mutations in the urine may substantially aid in the early detection of bladder cancer [29, 30]. However, exosomal TERC has thus far not been investigated as a diagnostic or prognostic biomarker for bladder cancer.

We found that TERC expression levels were not significantly different between tumor and adjacent nontumor tissues from BLCA patients but were significantly upregulated in urinary exosomes from BLCA patients compared with those from healthy controls. Furthermore, urinary exosomal TERC expression could distinguish between urinary benign diseases (inflammation, stones, and obstruction) and bladder cancer $(P<0.0001)$. TERC can circulate in whole body fluids under the envelopment of exosomes. Additionally, TERC could significantly distinguish high- versus low-grade BLCA $(P=0.0153)$, and Kaplan-Meier survival curves demonstrated its prognostic value. Importantly, multiple freeze-thaw cycles of urine did not affect the stability of exosomal TERC.

The exosome is the star molecule of liquid biopsy, and research into them is still in the early stages. Our findings are limited by current science and technology in terms of achieving rapid and efficient detection of exosomal RNA. To extract urinary exosomes, we employ the polyethylene glycol (PEG) precipitation technique, which normally involves around 10 hours of pretreatment (centrifugation and resting) and 1 hour of centrifugation at $10000 \times g$, and the exosome precipitation will be achieved using a standard high-speed centrifuge. Although costly ultracentrifuges are not needed, they still have the drawback of being timeconsuming and multistep. At this stage, urine exosomal TERC can only be used as an adjunct to cystoscopy, with diagnostic efficacy superior to existing NMP-22, BTA, and other indicators, allowing for patient review and follow-up. From experimental research to clinical application, not only medical but also engineering, science, and other interdisciplinary collaborations are required, and completely automated batch detection of urine exosomal TERC is still a long way off.

In the future, more data on the detection of urine exosomal TERC will be collected via multicenter and large sample studies, and patients will be followed for a longer period of time to validate its diagnostic and prognostic value. 
DSS

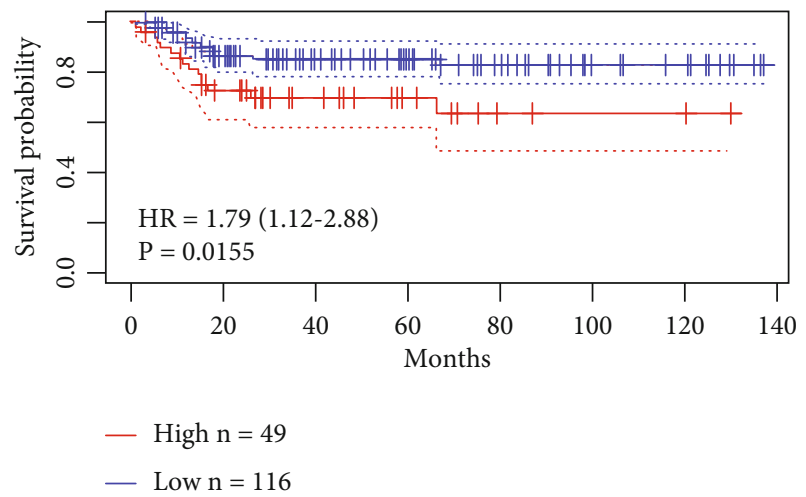

(a)
OS

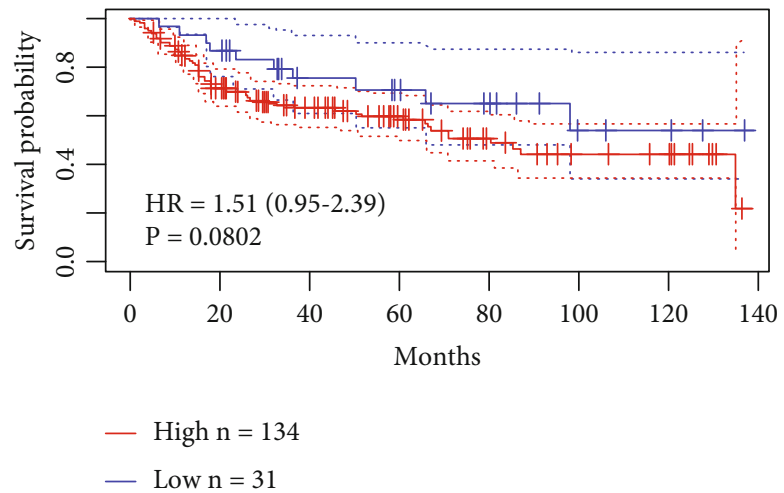

(b)

FIgURE 5: Analysis of Kaplan-Meier survival curves from the PrognoScan database. (a) The disease-specific survival (DSS) curve of TERC (hazard ratio [HR]: 1.79, 95\% confidence interval [CI]: 1.12-2.88, $P=0.0155)$. (b) The overall survival (OS) curve of TERC (HR: 1.51, 95\% CI: $0.95-2.39, P=0.0802)$. $\mathrm{HR}>1$ indicates a risk factor associated with poor patient outcomes.

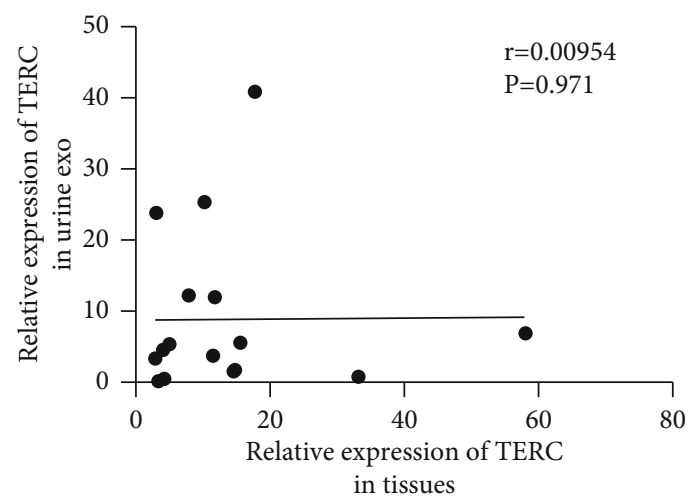

(a)

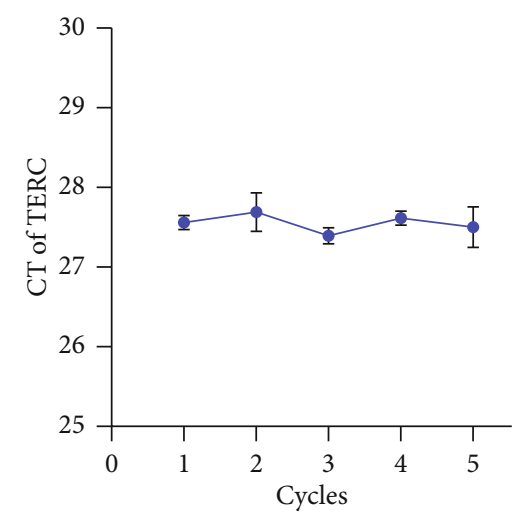

(b)

FIgURE 6: Correlation analysis of telomerase RNA component (TERC) levels in different sample types and its stability in exosomes. (a) TERC expression in tissues was uncorrelated with that in urine exosomes $(r=0.00954, P=0.971)$. (c) Exosomal TERC levels after one to five freeze-thaw cycles of urine samples. Results are presented as mean \pm SD.

\section{Conclusions}

In summary, the long noncoding RNA TERC was first found in urine exosomes in this article, and we demonstrated that urinary exosomal TERC can be used as a diagnostic and prognostic biomarker for BLCA with the added benefit of reducing unnecessary injuries from tissue biopsies. This study provides a convenient, stable, repeatable, and noninvasive index for clinicians and BLCA patients. Thus, our discovery will have profound implications for diagnosis and monitoring of bladder urothelial carcinoma.

\section{Data Availability}

The datasets during and/or analyzed during the current study are available from the corresponding authors on reasonable request.

\section{Conflicts of Interest}

The authors declare that there are no conflicts of interest regarding the publication of this paper.

\section{Acknowledgments}

The work was supported by the Department of Clinical Laboratory of Fudan University Shanghai Cancer Center, and I would like to show great gratitude to Prof. Lu Renquan for supplying us with information on many bladder cancer patients. This work was supported by the Science and Technology Innovation Action Plan of the Shanghai Science and Technology Commission (grant number 19411964800), the National Natural Science Foundation of China (grant numbers 81873975, 82072362, 82002222, 82002223), the Shanghai Public Health System Construction Three-Year Action Plan (2020-2022) Key Disciplines (grant number GWV- 
10.1-XK04), the Clinical Research Project of Tongji Hospital of Tongji University (grant number ITJ(ZD)1905, ITJ(QN)1905, ITJ(QN)2005), the Shanghai Post-Doctoral Excellence Program (grant number 2020409), and the Postdoctoral Science Foundation of China (grant numbers 2020M681399, 2020M681400).

\section{Supplementary Materials}

Supplementary 1. Supplementary File 1: the clinical information of patients.

Supplementary 2. Supplementary File 2: the absolute levels of genes and NMP-22 (ELISA).

\section{References}

[1] H. Sung, J. Ferlay, R. L. Siegel et al., "Global cancer statistics 2020: GLOBOCAN estimates of incidence and mortality worldwide for 36 cancers in 185 countries," CA: A Cancer Journal for Clinicians, vol. 71, no. 3, pp. 209-249, 2021.

[2] M. Zibelman, A. M. Asghar, D. C. Parker et al., "Cystoscopy and systematic bladder tissue sampling in predicting pT0 bladder cancer: a prospective trial," The Journal of Urology, vol. 205, no. 6, pp. 1605-1611, 2021.

[3] E. C. Hwang, H. S. Choi, S. I. Jung, D. D. Kwon, K. Park, and S. B. Ryu, "Use of the NMP22 BladderChek test in the diagnosis and follow-up of urothelial cancer: a cross-sectional study," Urology, vol. 77, no. 1, pp. 154-159, 2011.

[4] M. F. Sarosdy, R. W. deVere White, M. S. Soloway et al., "Results of a multicenter trial using the BTA test to monitor for and diagnose recurrent bladder cancer," The Journal of Urology, vol. 154, no. 2, pp. 379-384, 1995.

[5] R. Zhou, K. K. Chen, J. Zhang et al., "The decade of exosomal long RNA species: an emerging cancer antagonist," Molecular Cancer, vol. 17, no. 1, p. 75, 2018.

[6] D. M. Pegtel and S. J. Gould, "Exosomes," Annual Review of Biochemistry, vol. 88, pp. 487-514, 2019.

[7] L. E. Qu, J. Ding, C. Chen et al., "Exosome-transmitted lncARSR promotes sunitinib resistance in renal cancer by acting as a competing endogenous RNA," Cancer Cell, vol. 29, no. 5, pp. 653-668, 2016.

[8] B. Uszczynska-Ratajczak, J. Lagarde, A. Frankish, R. Guigó, and R. Johnson, "Towards a complete map of the human long non-coding RNA transcriptome," Nature Reviews. Genetics, vol. 19, no. 9, pp. 535-548, 2018.

[9] J. Cheng, J. Meng, L. Zhu, and Y. Peng, "Exosomal noncoding RNAs in glioma: biological functions and potential clinical applications," Molecular Cancer, vol. 19, no. 1, 2020.

[10] U. Gezer, E. Özgür, M. Cetinkaya, M. Isin, and N. Dalay, "Long non-coding RNAs with low expression levels in cells are enriched in secreted exosomes," Cell Biology International, vol. 38, no. 9, pp. 1076-1079, 2014.

[11] Y. Zhan, L. Du, L. Wang et al., "Expression signatures of exosomal long non-coding RNAs in urine serve as novel noninvasive biomarkers for diagnosis and recurrence prediction of bladder cancer," Molecular Cancer, vol. 17, no. 1, p. 142, 2018.

[12] H. J. Li, X. Gong, Z. K. Li et al., "Role of long non-coding RNAs on bladder cancer," Frontiers in Cell and Development Biology, vol. 9, article 672679, 2021.
[13] M. Xue, W. Chen, A. Xiang et al., "Hypoxic exosomes facilitate bladder tumor growth and development through transferring long non-coding RNA-UCA1," Molecular Cancer, vol. 16, no. $1,2017$.

[14] C. Chen, Y. Luo, W. He et al., "Exosomal long noncoding RNA LNMAT2 promotes lymphatic metastasis in bladder cancer," The Journal of Clinical Investigation, vol. 130, no. 1, pp. 404421, 2020.

[15] D. Chakravarti, K. A. LaBella, and R. A. DePinho, "Telomeres: history, health, and hallmarks of aging," Cell, vol. 184, no. 2, pp. 306-322, 2021.

[16] J. A. Baena-del Valle, Q. Zheng, D. M. Esopi et al., "MYC drives overexpression of telomerase RNA (hTR/TERC) in prostate cancer," The Journal of Pathology, vol. 244, no. 1, pp. 11-24, 2018.

[17] Y. Cheng, P. Liu, Q. Zheng et al., "Mitochondrial trafficking and processing of telomerase RNA TERC," Cell Reports, vol. 24, no. 10, pp. 2589-2595, 2018.

[18] M. L. Cayuela, J. M. Flores, and M. A. Blasco, "The telomerase RNA component Terc is required for the tumour-promoting effects of Tert overexpression," EMBO Reports, vol. 6, no. 3, pp. 268-274, 2005.

[19] H. Liu, Y. Yang, Y. Ge, J. Liu, and Y. Zhao, "TERC promotes cellular inflammatory response independent of telomerase," Nucleic Acids Research, vol. 47, no. 15, pp. 8084-8095, 2019.

[20] C. Théry, K. W. Witwer, E. Aikawa et al., "Minimal information for studies of extracellular vesicles 2018 (MISEV2018): a position statement of the International Society for Extracellular Vesicles and update of the MISEV2014 guidelines," Journal of extracellular vesicles, vol. 7 , no. 1, article 1535750, 2018.

[21] H. Zhou, P. S. Yuen, T. Pisitkun et al., "Collection, storage, preservation, and normalization of human urinary exosomes for biomarker discovery," Kidney International, vol. 69, no. 8, pp. 1471-1476, 2006.

[22] Y. Li, J. Zhao, S. Yu et al., "Extracellular vesicles long RNA sequencing reveals abundant mRNA, circRNA, and lncRNA in human blood as potential biomarkers for cancer diagnosis," Clinical Chemistry, vol. 65, no. 6, pp. 798-808, 2019.

[23] S. K. Tan, C. Pastori, C. Penas et al., "Serum long noncoding RNA HOTAIR as a novel diagnostic and prognostic biomarker in glioblastoma multiforme," Molecular Cancer, vol. 17, no. 1, p. 74, 2018.

[24] R. Zheng, M. Du, X. Wang et al., "Exosome-transmitted long non-coding RNA PTENP1 suppresses bladder cancer progression," Molecular Cancer, vol. 17, no. 1, 2018.

[25] M. Müller, H. Krause, R. Heicappell, J. Tischendorf, J. W. Shay, and K. Miller, "Comparison of human telomerase RNA and telomerase activity in urine for diagnosis of bladder cancer," Clinical cancer research, vol. 4, no. 8, pp. 1949-1954, 1998.

[26] L. Mezzasoma, C. Antognelli, C. del Buono et al., "Expression and biological-clinical significance of hTR, hTERT and CKS2 in washing fluids of patients with bladder cancer," BMC Urology, vol. 10, no. 1, 2010.

[27] N. I. Guidance, "Bladder cancer: diagnosis and management of bladder cancer," BJU international, vol. 120 , no. 6 , pp. $755-$ $765,2017$.

[28] L. M. Mikhaleva, V. V. Pechnikova, A. M. Pshikhachev et al., "Bladder cancer: update on risk factors, molecular and ultrastructural patterns," Current Medicinal Chemistry, vol. 28, 2021. 
[29] Y. Hayashi, K. Fujita, G. J. Netto, and N. Nonomura, "Clinical application of TERT promoter mutations in urothelial carcinoma," Frontiers in Oncology, vol. 11, article 705440, 2021.

[30] M. I. Hosen, N. Forey, G. Durand et al., "Development of sensitive droplet digital PCR assays for detecting urinary TERT promoter mutations as non-invasive biomarkers for detection of urothelial cancer," Cancers, vol. 12, no. 12, p. 3541, 2020. 